\title{
Inclusive production of jets and dijets at LHCb
}

\section{Giulio Auriemma* on behalf of the LHCb collaboration}

Universitá degli Studi della Basilicata, Italy and INFN Sezione di Roma,Italy

E-mail: giulio.auriemma@cern.ch

The region $\eta>3$ is a kinematic range of interest for Higgs searches, QCD physics and beyond the Standard Model studies in $p p$ interactions at $\mathrm{TeV}$ energies. We explore the feasibility of measuring jets in the LHCb experiment, mainly devoted to precision measurements in the $b$-physics domain, but covering the very forward region $2<\eta<4.5$. The jets reconstruction capabilities of LHCb are presented, together with some preliminary results on inclusive jets and dijets that show the potential interest of LHCb results for low- $x /$ high- $Q^{2}$ perturbative QCD tests. The data have been taken at LHC during the 2010 runs at $\sqrt{s}=7 \mathrm{TeV}$.

Kruger 2010: Workshop on Discovery Physics at the LHC

December 5 - 10, 2010

Kruger National Park, Mpumalanga, South Africa

\footnotetext{
${ }^{*}$ Speaker.
} 


\section{Introduction}

The LHCb detector [1] is a single arm spectrometer for the $2 \leq \eta \leq 5$ region, with excellent particle identification and charged particle tracking from vertex detector to calorimeters. Neutral pions are reconstructed using calorimeter clusters not associated to charged tracks. The detector is optimized for the reconstruction of the $b$-hadrons.

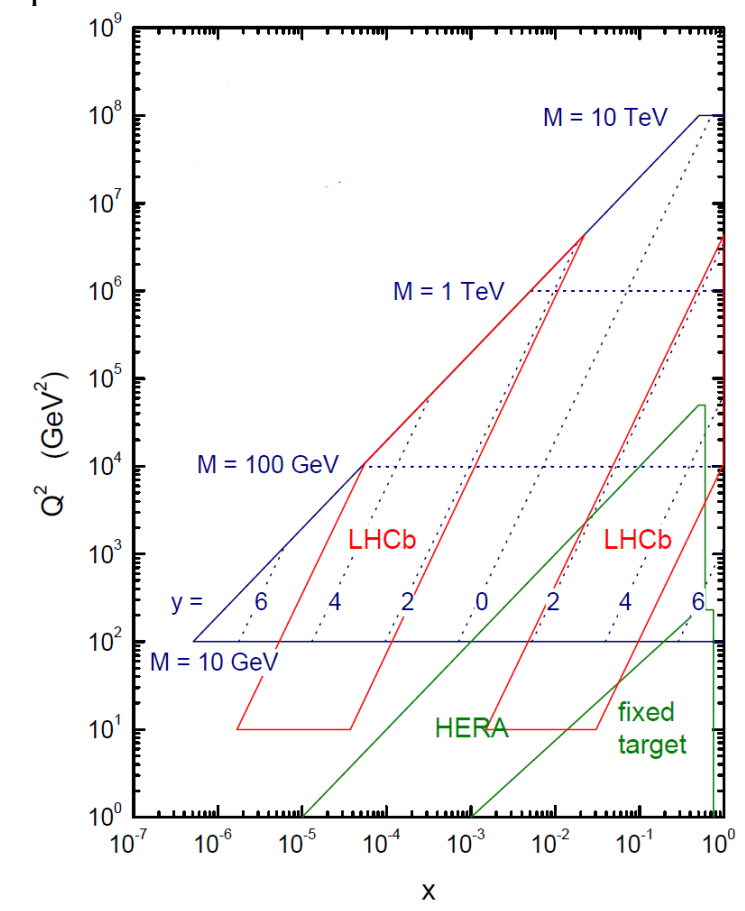

Figure 1: Region in the $\left(x, Q^{2}\right)$ plane accessible to LHCb (adapted from Ref. [4]).
Jets are fundamental tools for the study of any process that involves final state partons, either in the Standard Model (SM) or beyond it [2]. Beside the expected discovery of new particles and/or processes, jet physics in the forward region $\eta>3$ at LHC (not covered by experiments at Tevatron) could open new opportunities for testing some specific aspects of perturbative QCD, as for example the Parton Distribution Functions (PDF) and their dynamical evolution in the proton [3].

Figure 1 (adapted from Ref. [4]) shows the region accessible to $\mathrm{LHCb}$ in the Bjorken invariants $\left(x, Q^{2}\right)$ plane, where $x$ is the fraction of the beam momentum carried by the colliding parton and $Q^{2}$ the squared momentum transfer in the partonic collision. Inclusive jet events $p p \rightarrow$ jet $+X$ and dijet events $p p \rightarrow j^{2 e t_{1}}+\mathrm{jet}_{2}+X$ in LHCb can provide valuable information on the PDF in a regime of $x<10^{-3}$. This region has been already explored by H1 and ZEUS at HERA [5] at $Q^{2}<10$ $\mathrm{GeV}^{2}$. Figure 1 shows that new data in the region $x<10^{-3}$ and $Q^{2}>100 \mathrm{GeV}^{2}$ can test effectively the evolution of the PDF with $Q^{2}$ [4].

This preliminary analysis investigates the performance of jet detection at LHCb and establishes a benchmark for future more quantitative analysis. To do this we have exploited the low luminosity runs of the LHC commissioning phase (May-August 2010).

\section{Data, event selection and jet reconstruction}

Different $\mathrm{LHCb}$ sub-detectors are used in this analysis. The tracking system placed along the beam line of the LHC, starting with the Vertex Locator (VELO), that provides precise locations for primary $p p$ interaction vertices and contributes to the measurement of track momentum. The Electromagnetic Calorimeter (ECAL) used for $\pi^{0}$ detection and the Hadron Calorimeter (HCAL) which essentially provides the hardware trigger for jet events. These last two detectors, in combination with the $4 \mathrm{Tm}$ dipole magnet, are used to measure the momenta of charged particles.

$\mathrm{LHCb}$ at present does not have a dedicated jet trigger. The jet reconstruction has been applied to hardware triggered events, eventually prescaled when required by the production rate $[1,6]$. 

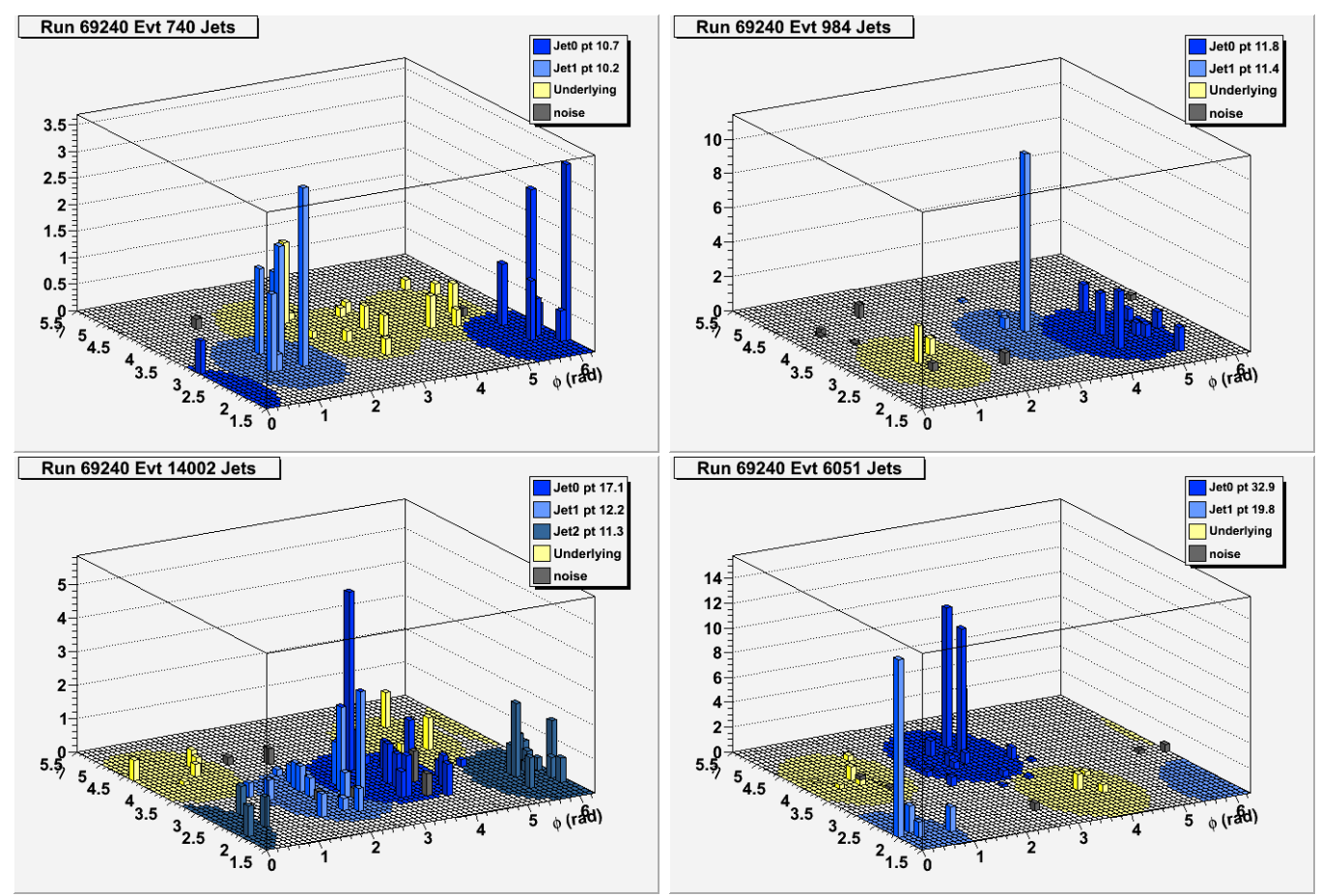

Figure 2: Some illustrative events reconstructed in $\mathrm{LHCb}$ where the leading jet has $p_{T}^{\text {jet }}>10 \mathrm{GeV} / \mathrm{c}$ from very early runs at $7 \mathrm{TeV}$. One of the runs on which we have tested the selection criteria. Jets reconstruction has been done with the $k_{T}$ algorithm in the E-recombination scheme with $R=1$ and $p_{T}^{\text {cut }}=1 \mathrm{GeV} / \mathrm{c}$. As "Underlying Event" (yellow) we classify any particle non clustered in the two jets with higher momentum, while "noise" is any track with $\chi^{2} / d o f \geq 10$.

Two periods of data taking in the year 2010 are included in this analysis: i) from May, 10 to July, $5\left(50 \mathrm{nb}^{-1}\right)$ with a trigger requiring at least one hadron with $E_{T}>0.24 \mathrm{GeV}$; ii) from July, 12 to August, $24\left(0.97 \mathrm{pb}^{-1}\right)$ with a sligthly harder trigger required for the hadron $E_{T}>2.26 \mathrm{GeV}$.

Events included in the analysis are required to have only one reconstructed primary vertex and a minimum of five charged tracks with $p_{T} \geq 0.2 \mathrm{GeV} / \mathrm{c}$. Exclusion of events with more than one reconstructed primary vertex has been introduced in order to get a pure sample of jets coming from a single $p p$ interaction. This requirement reduces the statistics by a factor $\sim 2$.

Jets clustering has been performed with the $k_{T}$ algorithm [7], as implemented in the FastJet package [8] using as inputs the four-momenta of the particles, assumed to be massless. This is similar to the "particle flow jets" (PFJ) type of reconstruction applied by the CMS experiment [9]. The uncorrected four-momentum $\left\{E^{R A W}, \vec{p}^{R A W}\right\}$ of the jet is calculated in the E-recombination scheme, namely as the sum of the four-momenta of its constituents. In this preliminary analysis no corrections for jet energy scale, acceptance and smearing have been applied.

Figure 2 shows examples of the jet events detected in the very early runs of LHCb at $\sqrt{s}=7$ $\mathrm{TeV}$, with a leading jet $p_{T}^{R A W} \geq 10 \mathrm{GeV} / \mathrm{c}$. There is a clear observation of dijets in the LHCb acceptance. 


\section{Results}

Figure 3 shows the differential $E_{T}^{R A W}$ distribution of inclusive jet events, uncorrected for JES, acceptance and efficiencies, defined as $E_{T}^{R A W}=\sum E_{j} / \cosh \eta_{j e t}$ where $E_{j}$ are the energies of the constituent particles of the jet and $\eta_{j e t}=-\ln \tan \theta / 2$ its pseudorapidity in the laboratory frame. In this figure the experimental distribution is compared with that obtained applying the $k_{T}$ algorithm to particles simulated with the LHCb MonteCarlo program [10] based upon PYTHIA 6.4 [11] with CTEQ6-LO parton distribution functions [12], tracked through the detector with the GEANT4 package [13], taking into account the details of the geometry and ma-

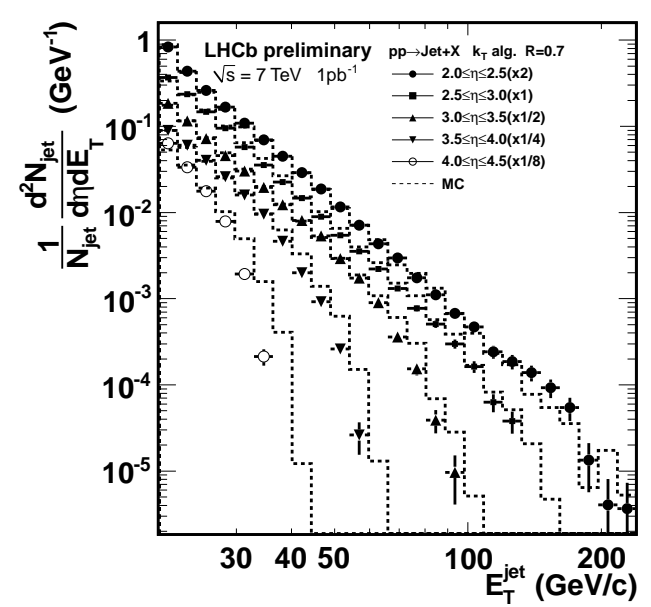

Figure 3: Normalized differential distribution for inclusive jets compared with $\mathrm{LHCb}$ MonteCarlo program (see text). terial composition of the $\mathrm{LHCb}$ detector.

Figure 4 shows the azimuthal angle difference $\Delta \phi=\phi_{j e t_{1}}-\phi_{j e t_{2}}$ for the two jets with higher $p_{T}^{R A W}$ in all the events that have at least two jets with $p_{T}^{R A W}>20 \mathrm{GeV} / \mathrm{c}$. It clearly illustrates that a large number of jet pairs with $\Delta \phi \approx \pi$ is present in the sample. The distribution of Fig. 4 is reasonably well fitted by a Gaussian peak plus a polynomial background, likely originated by the accidental pairing of a partonic jet with a jet from the underlying event.
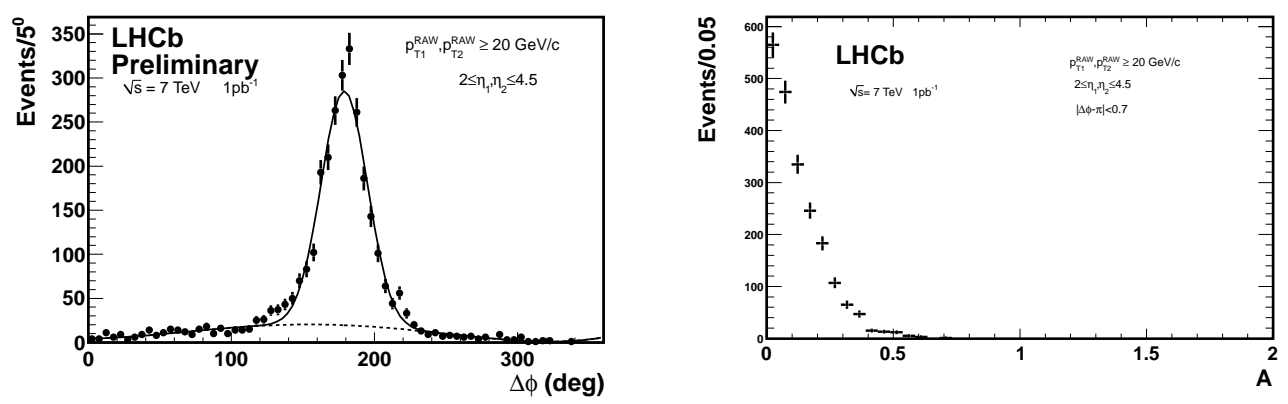

Figure 4: Panel (a) shows the distribution of the azimuthal angle difference $\Delta \phi$ of the two leading jets. The full line is the result of the fit of the data with the sum of a polynomial background (dashed line) and a Gaussian function. Panel (b) shows the differential distribution of the asymmetry parameter $A$, defined in the text.

Figure $4 \mathrm{~b}$ shows the differential distribution of the asymmetry parameter, defined as

$$
A=\frac{\left|p_{T 1}^{R A W}-p_{T 2}^{R A W}\right|}{p_{T 1}^{R A W}+p_{T 2}^{R A W}} .
$$

It is worth noting that this parameter is not changed by the jet energy scale correction if $p_{T 1}^{R A W} \simeq$ $p_{T 2}^{R A W}$. 
The salient features of Figs. $4 \mathrm{a}$ and $4 \mathrm{~b}$ are indicative of the presence of long range QCD effects, such as the initial and final state radiation and the multiple partonic interactions.

The invariant mass of the dijet system, not corrected for jet energy scale, is defined as

$$
\tilde{M}_{j j}=\sqrt{\left(E_{1}^{R A W}+E_{2}^{R A W}\right)^{2}-\left(\vec{p}_{1}^{R A W}+\vec{p}_{2}^{R A W}\right)^{2}}
$$

where $\left\{E_{1}^{R A W}, \vec{p}_{1}^{R A W}\right\}$ and $\left\{E_{2}^{R A W}, \vec{p}_{2}^{R A W}\right\}$ are respectively the measured four-momenta of the two jets. Fig. 5a shows its distribution for jet pairs satisfying $|\Delta \phi-\pi| \leq 40^{\circ}$ and $A \leq 0.2$ that is well described by the LHCb Monte Carlo.
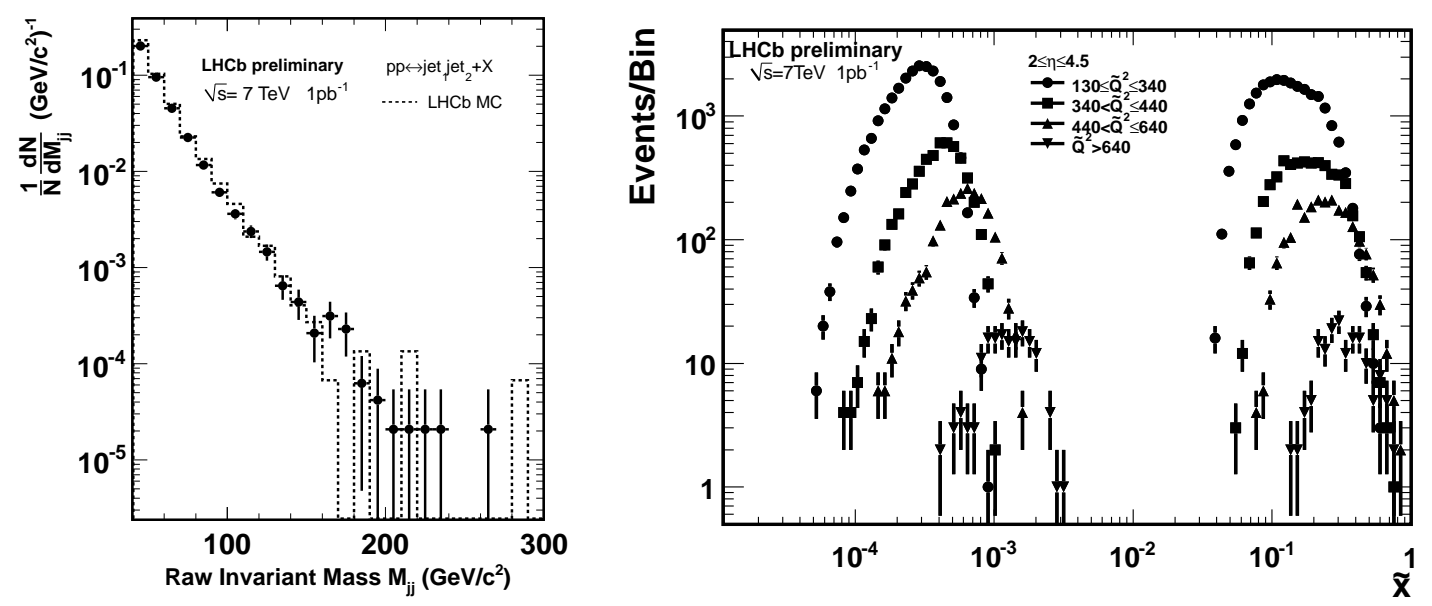

Figure 5: Panel (a) shows the normalized invariant mass distribution for jet pairs with $p_{T 1}$ and $p_{T 2} \geq$ $20 \mathrm{GeV} / \mathrm{c},|\Delta \phi-\pi| \leq 40^{\circ}$ and $A \leq 0.2$. The dashed lines shows the LHCb-MC simulated distribution (see text). Panel (b) shows the uncorrected dijets $\tilde{x}$ distribution separated into $\tilde{Q}^{2}$ bins.

The kinematic of the scattering of light partons is determined by the fraction of momentum carried by the two incoming partons $x_{1}$ and $x_{2}$ and the $Q^{2}$. From the measurement of the two jets uncorrected four-momenta are obtained the quantities:

$$
\tilde{x}_{1,2}=\frac{2 p_{T 1,2}^{R A W}}{\sqrt{s}} e^{ \pm \bar{\eta}} \cosh \frac{\Delta \eta}{2} \quad \tilde{Q}^{2}=p_{T 1}^{R A W} p_{T 2}^{R A W}
$$

where $p_{T 1}^{R A W}$ and $p_{T 2}^{R A W}$ are respectively the uncorrected transverse momentum of the two jets, $\bar{\eta}=$ $\frac{1}{2}\left(\eta_{1}+\eta_{2}\right)$ the pseudo-rapidity of the center-of-mass of colliding partons, $\Delta \eta=\eta_{1}-\eta_{2}$ the rapidity gap and $\sqrt{s}=7 \mathrm{TeV}$ the square of the center-of-mass energy of the $p p$ collision. The two quantities $\left(\tilde{x}, \tilde{Q}^{2}\right)$ are lower estimate of the Bjorken invariants $\left(x, Q^{2}\right)$. Finally Fig. $5 \mathrm{~b}$ shows the uncorrected $\tilde{x}$ distribution for $\tilde{Q}^{2}$ bins. Ultimately the calibrated data could be used to constraint the PDF at $x<10^{-3}$ and $Q^{2}>100 \mathrm{GeV}^{2}$.

\section{Conclusion}

This preliminary analysis of $\sim 1 \mathrm{pb}^{-1}$ of LHCb data shows that the inclusive jets distribution and dijets characteristics can be measured by the LHCb experiment in the range $2 \leq \eta \leq 4.5$. 
Interesting results on perturbative QCD at $x<10^{-3}$ are expected from the $\sim 36 \mathrm{pb}^{-1}$ data obtained in the 2010 runs of the LHC.

\section{References}

[1] A. A. Alves et al., (The LHCb collaboration), The LHCb Detector at the LHC, JINST 3 (2008) S08005; O. Steinkamp, LHCb Status, First Physics and Discovery Potential, in these procedings PoS (Kruger 2010) 024.

[2] A. Ahmed and G. Kramer, Jets in QCD, Eur. Phys. J. H in press [arXiv:1012.2288v1]; G. P. Salam, Towards Jetography, Eur. Phys. J., C67 (2010) 637-686.

[3] F. Hautmann, Forward jets in hadron-hadron collisions, Talks given at QCD 2010, Montpellier, June 2010 [arXiv:1101.2656]; D. G. d'Enterria Forward jets physics in ATLAS, CMS and $L H C b$, invited contribution EDS'09 Int. Conf. (CERN, June-July 2009)[arXiv:0911.1273v1].

[4] R. S. Thorne, A. D. Martin, W. J. Stirling, and G. Watt, Parton Distributions and QCD at LHCb, Proc. of DIS2008, April 7-11, 2008 London, England [arXiv:0808.1847].

[5] J. Baines et al., Heavy quarks, Summary report for the HERA - LHC Workshop 2006 [arXiv:hep-ph/0601164v2].

[6] E. Van Herwijnen for the LHCb collaboration The LHCb Trigger, talk at ICHEP2010 July 2010, Paris, [CERN-LHCb-PROC-2010-055].

[7] S. Catani, Y. L. Dokshitzer, M. H. Seymour, and B. R. Webber, Longitudinally invariant $k_{t}$ clustering algorithms for hadron hadron collisions, Nucl. Phys. B406 (1993) 187-224; S. D. Ellis and D. E. Soper, Successive combination jet algorithm for hadron collisions, Phys. Rev. D48 (1993) 3160-3166.

[8] M. Cacciari and G. P. Salam, ”Dispelling the $N^{3}$ myth for the $k_{t}$ jet-finder”, Phys. Lett. B641 (2006) 57-61.

[9] S. Salur for the CMS collaboration, Jet Results and Jet Reconstruction Techniques in $p+p$ and their Prospects in Pb+Pb Collisions in CMS, Presented at JET2010, [CERN-CMS-CR2010-162].

[10] I. Belyaev et al., "Handling of the generation of primary events in Gauss, the LHCb simulation framework”, Proc. of NSS2010, Knoxville, October 2010, [CERN-LHCb-PROC-2010056].

[11] T. Sjostrand, S. Mrenna, and P. Z. Skands, PYTHIA 6.4 Physics and Manual, JHEP 5 (2006) 026 [hep-ph/0603175].

[12] P. M. Nadolsky et al., Implications of CTEQ global analysis for collider observables, Phys. Rev. D78 (2008) 013004.

[13] S. Agostinelli et al., "GEANT 4-a simulation toolkit”, Nucl. Instrum. and Methods A506 (2003) $250-303$. 